Received: 2017.07.12

Accepted: 2018.01.26

Published: 2018.07 .26

Authors' Contribution:

Study Design A

Data Collection B

Statistical Analysis C

Data Interpretation D

Manuscript Preparation E

Literature Search F

Funds Collection G

Corresponding Author:

Source of support:

\title{
Upregulation of CXCR7 Is Associated with Poor Prognosis of Prostate Cancer
}

\author{
CDE 1 Jihua Yang* \\ ABC 2 Hao Tang* \\ CDE 3 Jingyu Huang* \\ ABFG 4 Huaijie An
}

1 Department of Oncology, Naval General Hospital, Beijing, P.R. China 2 Department of Urology, Jinling Hospital, Nanjing, Jiangsu, P.R. China

3 Department of Thoracic and Cardiovascular Surgery, Zhongnan Hospital of Wuhan University, Wuhan, Hubei, P.R. China

4 Central Laboratory, Naval General Hospital, Beijing, P.R. China

Background: Prostate cancer (PCa) is a prevalent cancer in males. $C X C R 7$ exhibits oncogenic actions in various cancers. The aim of our study was to explore the clinical significance of $C X C R 7$ in patients with $\mathrm{PCa}$.

Material/Methods:

* Co-first authors; Jihua Yang, Hao Tang, Jingyu Huang

Huaijie An, e-mail: j990jsg@126.com

Funding was provided by the Natural Science Foundation of Jiangsu Province (Grant No. BK20140735)

Results: $\quad C X C R 7$ was upregulated in PCa tissues $(P<0.05)$ and was correlated with PSA $(P=0.023)$, differentiation $(P=0.022)$, and lymph node metastasis $(P=0.018)$. The results of MTT and Transwell assays demonstrated that inhibition of $C X C R 7$ suppressed PCa cells growth and migration. Additionally, high $C X C R 7$ level predicted poor overall survival (log rank test, $P=0.019)$. CXCR7 was a valuable prognostic biomarker for PCa patients ( $H R=2.271$, $95 \% \mathrm{Cl}=1.093-4.719, P=0.028)$.

Conclusions: $\quad C X C R 7$ is an oncogene in $\mathrm{PCa}$ that can promote aggressive progression of $\mathrm{PCa}$ through enhancing proliferation and migration of the tumor cells. CXCR7 is an independent biomarker for the prognosis of PCa.

MeSH Keywords: Genes, vif • Prognosis • Prostatic Neoplasms

Full-text PDF: https://www.medscimonit.com/abstract/index/idArt/906180

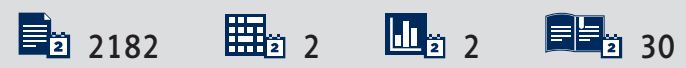




\section{Background}

Prostate cancer $(\mathrm{PCa})$ is a malignant tumor among men and is a leading cause of cancer-related deaths worldwide [1,2]. The traditional treatments for $\mathrm{PCa}$ include surgery, chemoradiotherapy, and biotherapy. The therapeutic effects are satisfactory for localized PCa; however, a subgroup of patients develop to recurrence or metastasis, resulting in therapy failures and dismal prognosis [3]. Effective biomarkers are pivotal for early diagnosis and appropriate treatments. The commonly used biomarker for PCa is PSA (prostate-specific antigen), but its low specificity may contribute to over-diagnosis and over-treatment [4]. Thus, it is urgent to find novel and effective biomark ers to predict prognosis and guide therapy for PCa patients.

Chemokines are low molecular weight proteins secreted by bone marrow stromal cells and other related mesothelial and epithelial cells. According to their conserved cysteine, chemokines can be divided into 4 groups: CXC, CC, C, and CX3C family [5]. Cooperating with corresponding receptors, chemokines exert proinflammatory properties and play important roles in chemotaxis and metabolism of leukocytes [6]. Abnormal expression of chemokines can contribute to various diseases and cancers through regulating development, angiogenesis, and atherosclerosis $[7,8]$. $C X C R 7$ is newly identified as a receptor of CSCL12. CXCR7 belongs to the G-protein-coupled receptor family and consists of 362 amino acids. CXCR7 gene is located in the human chromosome 2 q37. Growing evidence shows that $C X C R 7$ plays an important role in the development of tumors. Upregulation of $C X C R 7$ serves as an oncogene in various of cancers, such as breast cancer, lung cancer, and glioma $[9,10]$. The oncogenetic action of $C X C R 7$ in PCa was also reported in previous research. Singh et al. reported that inducing $C X C R 7$ high expression in normal PCa cells can enhance its proliferative ability [11]. However, the predictive role of $C X C R 7$ in PCa prognosis remains unclear.

In the present study, we aimed to explore the expression profile of $C X C R 7$ in PCa tissues, as well as its association with clinicopathologic characteristics of patients. MTT and Transwell assays were carried out to investigate the functions of $C X C R 7$ on biological behaviors of PCa cells. In addition, we evaluated the prognostic significance of $C X C R 7$ in PCa patients. Our results may provide a novel indicator for $\mathrm{PCa}$.

\section{Material and Methods}

\section{PCa tissue samples collection}

We obtained 152 pairs of PCa tissues and adjacent normal tissue samples during curative resection of PCa patients at Naval General Hospital. None of the patients enrolled in our study had received chemo- or radiotherapy before surgery. After collection, all tissue samples were immediately frozen in liquid nitrogen and then kept at $-80^{\circ} \mathrm{C}$ for further use. The PCa tissues were confirmed by 2 clinical pathologists based on immunohistochemical analysis results. The adjacent normal tissues were defined as those $>3 \mathrm{~cm}$ away from the cancer tissues. The extent of PCa aggressiveness was evaluated according to the TNM staging system (AJCC Cancer Staging Manual) [12]. The differentiation is the mature degree of tumor tissue. The differentiation degrees of the PCa tissues were estimated using Gleason score. Patients with Gleason score less than 7 were defined as high differentiation, Gleason score 7 was defined as moderate differentiation, while Gleason score $>7$ was defined as low differentiation [13]. The study was approved by the hospital Ethics Committee and we obtained the written informed consent from all patients in advance.

All the PCa patients in our study had participated in a postoperative 5-year follow-up investigation. The PCa patients were followed up every 3 months for the first year and then every 6 months for the subsequent 2 years, and annually for the last 2 years. The clinicopathological features are listed in Table 1, including age, tumor size, PSA, TNM stage, differentiation, and lymph node metastasis.

\section{Real time PCR}

Total RNA was extracted from the tissues using Trizol reagent (Invitrogen). RNA concentration and purity were measured using a Nano-Drop ND-1000 spectrophotometer (NanoDrop Technologies, Houston, TX, USA). cDNA was synthesized from total RNA using the ReverTra Ace- $\alpha$ First-Strand cDNA Synthesis Kit (Toyobo (Shanghai) Biotech Co., Ltd., Shanghai, China). qRT-PCR was performed using the $A B I$ Prism 7900 Sequence Detection System (Applied Biosystems, Foster City, CA, USA) with SYBR Green Premix Ex Taq (Takara, Dalian, China). $\beta$-actin served as internal control. The primer sequences of $C X C R 7$ and $\beta$-actin were as follows: CXCR7: 5'-CTATGACACGCACTGCTACATC-3' (forward), 5'-CTGCACGAGACTGACCACC-3' (reverse); $\beta$-actin 5'-ATGGAGGGGAATACAGCCC-3' (forward), 5'-TTCTTTGCA GCTCCTTCGTT-3' (reverse) [14]. We measured the relative expression of $C X C R 7$ by normalizing with $\beta$-actin and using the $2^{-\Delta \Delta c t}$ method. Each measurement was performed in triplicate.

\section{Cell culture and transfection}

Human PCa cell line PC3 was purchased from the Central Laboratory of the Affiliated Hospital of Qingdao University (Qingdao, Shandong Province, China). DMEM medium was used to culture the PC3 cell lines and the medium was supplemented with 10\% FBS (fetal bovine serum), $1 \%$ glutamine, $100 \mathrm{U} / \mathrm{ml}$ of penicillin, and $100 \mu \mathrm{g} / \mathrm{ml}$ of streptomycin, at $37^{\circ} \mathrm{C}$ with $5 \% \mathrm{CO}_{2}$. 
Table 1. Association of $C X C R 7$ expression with clinicopathological features of prostate cancer patients.

\begin{tabular}{|c|c|c|c|c|c|}
\hline \multirow{2}{*}{ Characteristics } & \multirow{2}{*}{$N=152$} & \multicolumn{2}{|c|}{ CXCR7 expression } & \multirow{2}{*}{$\chi^{2}$} & \multirow{2}{*}{$P$ values } \\
\hline & & Low $(n=61)$ & High (n=91) & & \\
\hline \multicolumn{6}{|l|}{ Age (years) } \\
\hline$\geq 65$ & 88 & 35 & 53 & \multirow{2}{*}{0.011} & \multirow{2}{*}{0.916} \\
\hline$<65$ & 64 & 26 & 38 & & \\
\hline \multicolumn{6}{|l|}{ Tumor size $(\mathrm{cm})$} \\
\hline$\geq 3$ & 79 & 30 & 49 & \multirow{2}{*}{0.319} & \multirow{2}{*}{0.573} \\
\hline$<3$ & 73 & 31 & 42 & & \\
\hline \multicolumn{6}{|l|}{ PSA (ng/ml) } \\
\hline$\leq 20$ & 112 & 51 & 61 & \multirow{2}{*}{5.173} & \multirow{2}{*}{0.023} \\
\hline$>20$ & 40 & 10 & 30 & & \\
\hline \multicolumn{6}{|l|}{ Differentiation } \\
\hline Well+moderate & 109 & 50 & 59 & \multirow{2}{*}{5.284} & \multirow{2}{*}{0.022} \\
\hline Poor & 43 & 11 & 32 & & \\
\hline \multicolumn{6}{|l|}{ TNM stage } \\
\hline $1+I 1$ & 81 & 37 & 44 & \multirow{2}{*}{2.221} & \multirow{2}{*}{0.136} \\
\hline III+IV & 71 & 24 & 47 & & \\
\hline \multicolumn{6}{|c|}{ Lymph node metastasis } \\
\hline Negative & 87 & 42 & 45 & \multirow{2}{*}{5.617} & \multirow{2}{*}{0.018} \\
\hline Positive & 65 & 19 & 46 & & \\
\hline
\end{tabular}

PC3 cells at logarithmic growth phase were inoculated in a 96-well plate until the attachment efficiency reached 40-60\%. Then, the cells were transfected with CXCR7-siRNA using Lipofectamine 2000 Reagent according to the manufacturer's instructions. At $6 \mathrm{~h}$ after transfection, we continued to incubate the cells in 10\% FBS DMEM medium. Then, cells were harvested for proliferation and migration analyses after culturing for $48 \mathrm{~h}$. The cells transfected with CXCR7-NC (Applied Shanghai GenePharma Co., Ltd) were used as controls.

\section{MTT assay}

To determine the effect of $C X C R 7$ on the proliferation of PC3 cells, MTT assay was performed. The transfected cells were seeded into 96-well plates with $200 \mu \mathrm{l}$ per well (about $10^{4}$ cells/well) and cultured for $3-5$ days at $37^{\circ} \mathrm{C}$ in a $5 \% \mathrm{CO}_{2}$ incubator. MTT assay was performed at $24 \mathrm{~h}, 48 \mathrm{~h}$, and $72 \mathrm{~h}$. We added $20 \mu \mathrm{l}$ MTT (Sigma, St. Louis, MO, USA) to each well at a final concentration of $0.2 \mathrm{mg} / \mathrm{ml}$, and incubated for $4 \mathrm{~h}$ continuously, and then resolved with $150 \mu$ DMSO (Sigma) with low-speed shock for $10 \mathrm{~min}$. The OD values were measured at $490 \mathrm{~nm}$ using a Thermo Scientific Microplate Absorbance Reader (Thermo Biotechnology, USA). Each detection was performed in triplicate.

\section{Transwell migration assay}

We used Transwell assay to detect the migratory ability of PC3 cells transfected with CXCR7 siRNA. The cells (about $10^{4}$ cells/ well) were re-suspended in $200 \mu \mathrm{l}$ serum-free medium and seeded on the top chamber of a Transwell ${ }^{\circledR}$ device (6.5-mm Transwell filter with 8.0- $\mu \mathrm{m}$ pore polycarbonate membrane). Then, we added $600 \mu \mathrm{l}$ medium containing $10 \%$ FBS on the bottom chamber. After incubation for $24 \mathrm{~h}$ at $37^{\circ} \mathrm{C}$ with $5 \%$ $\mathrm{CO}_{2}$, we fixed the cells attached to the lower surface of the membrane with $4 \%$ paraformaldehyde at room temperature for $30 \mathrm{~min}$ and stained them with crystal violet (Beyotime Institute of Biotechnology, China). The number migrating cells was calculated from photomicrographs. Each group was assessed at least 3 times.

\section{Statistical analysis}

SPSS 19.0 software was used for data analysis and GraphPad Prism 5 was used for plotting graphs. All data are expressed as mean \pm SD. The difference of $C X C R 7$ expression between groups was analyzed by $t$ test. The relationship between $C X C R 7$ expression and clinical features of PCa patients was analyzed with the chi-square test. Survival curves were constructed using Kaplan-Meier method and compared by log rank test. Cox 
A

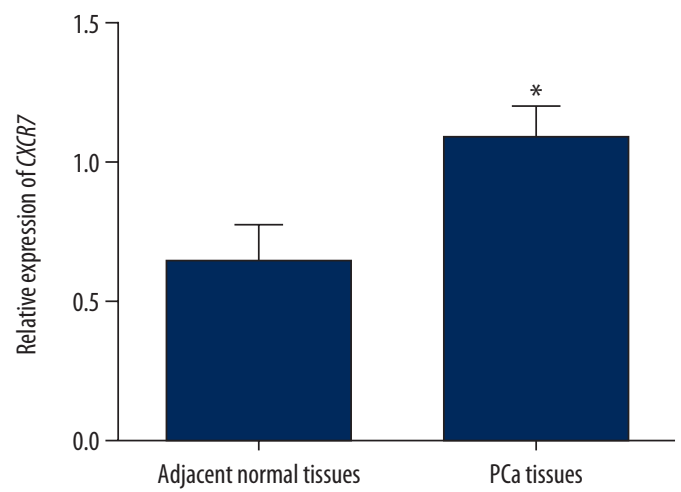

C

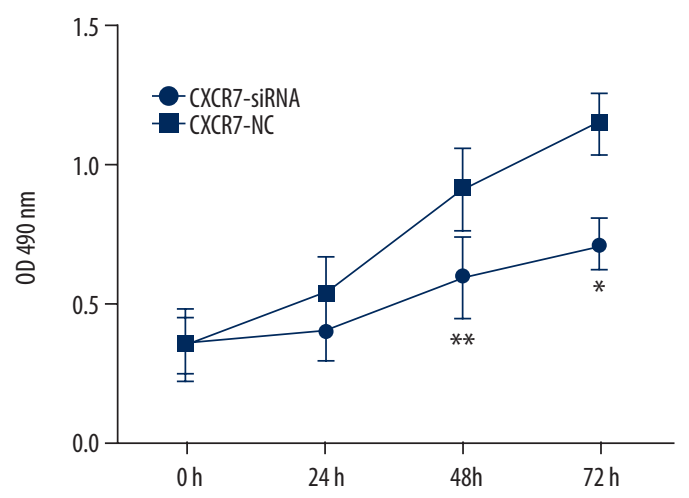

B

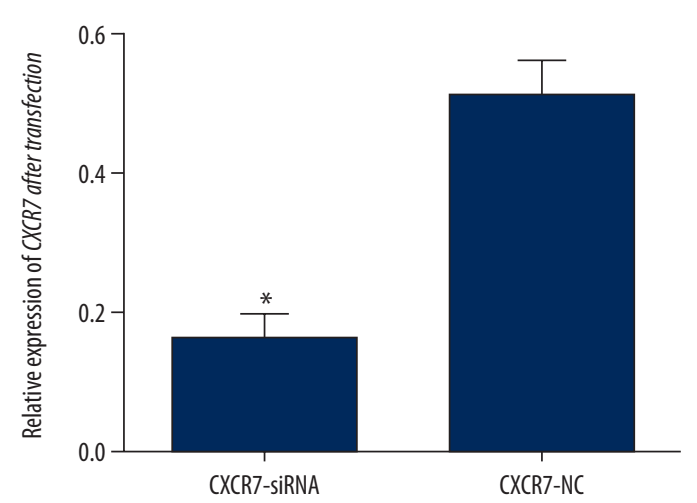

D

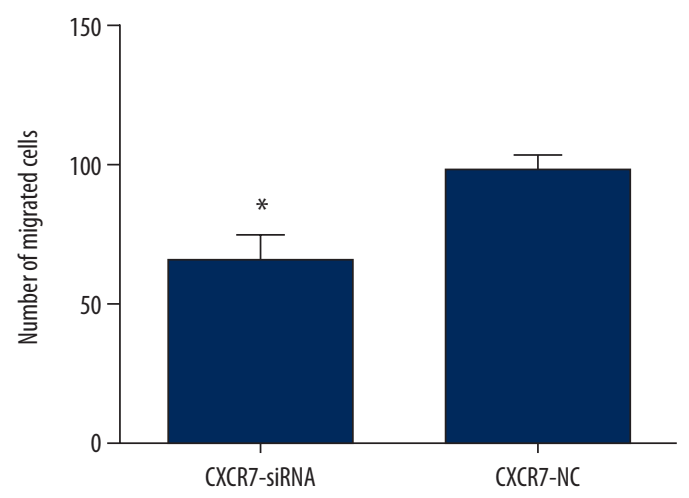

Figure 1. The expression pattern of $C X C R 7$ in tissues specimens, and its effects on the proliferation and migration of PCa cells in vitro. (A) Expression level of $C X C R 7$ between $P C$ a tissues and adjacent normal tissues. The results suggested that $C X C R 7$ appeared to be highly expressed in PCa tissues compared with non-cancerous tissues $(P<0.05)$. (B) $C X C R 7$ expression after transfected with CXCR7-siRNA and CXCR7-NC. CXCR7-siRNA successfully inhibited the expression of CXCR7 in PC3 cells $(P<0.05)$. (C) The proliferation of $P C 3$ cell line after transfection. Inhibition of $C X C R 7$ expression suppressed the proliferative ability of PC3 cells. (D) The migration ability of PC3 cell line after transfection. Analysis indicated that PC3 cells transfected with CXCR7siRNA had decreased migratory ability compared with those transfected with empty vector $(P<0.05)$.

regression analysis was used for the univariate and multivariate analyses. Differences were considered statistically significant at $P<0.05$.

\section{Results}

\section{High expression of CXCR7 in PCa}

We used qRT-PCR to examine the expression of $C X C R 7$ in 152 pairs of PCa tissues and adjacent normal tissues. The results showed that $C X C R 7$ expression in PCa tissues was significantly higher than that in adjacent normal tissues $(P<0.05)$ (Figure $1 \mathrm{~A})$.

\section{CXCR7 promoted the proliferation and migration of $\mathrm{PCa}$ cells}

To explore the effect of $C X C R 7$ on behaviors of PCa cells, CXCR7siRNA was constructed. The results shown in Figure $1 \mathrm{~B}$ demonstrate that the siRNA vector was successfully constructed. MTT analysis indicated that the growth of cells transfected with CXCR7-siRNA was significantly decreased compared with the CXCR7-NC group $(P<0.05)$ (Figure 1C). As shown in Figure 1D, the migratory ability of $C X C R 7$-siRNA-transfected cells was reduced compared with the CXCR7-NC group $(P<0.05)$.

\section{The relationship between $C X C R 7$ expression and clinicopathologic features of PCa}

We also explored the relationship between $C X C R 7$ expression level and clinicopathologic characteristics in PCa patients. The patients were grouped into high-expression and low-expression 


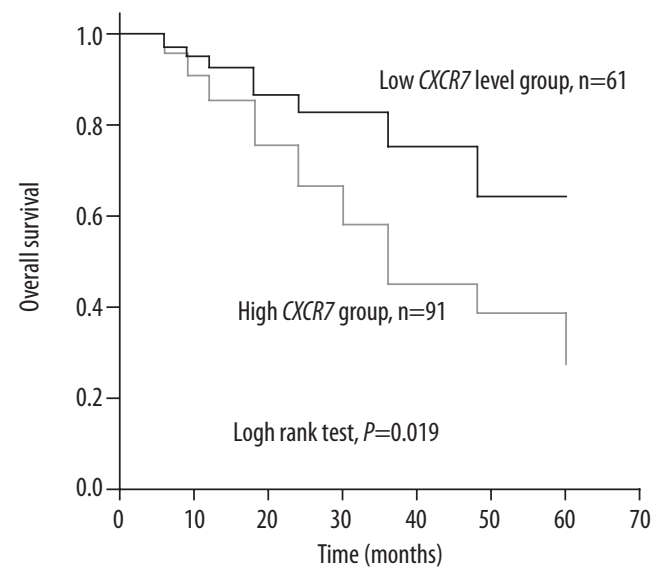

Figure 2. Kaplan-Meier analysis of PCa patients based on their expression of $C X C R 7$. The overall survival curve demonstrated that patients with high level of $C X C R 7$ had a significantly worse survival than those with low level (log rank test, $P=0.019$ ).

groups according to their median expression value of $C X C R 7$. The results in Table 1 show that $C X C R 7$ expression was obviously associated with PSA ( $P=0.023)$, differentiation $(P=0.022)$, and lymph node metastasis $(P=0.018)$. However, no obvious relationship was found between $C X C R 7$ expression and the age, tumor size, or TNM stage of the patients (all $P>0.05$ ).

\section{Correlation between CXCR7 expression and overall survival of $\mathrm{PCa}$ patients}

The relationship between the expression of $C X C R 7$ level and survival time of the PCa patients was evaluated by KaplanMeier analysis. The 5-year survival rate for PCa patients with high $C X C R 7$ expression was significantly lower than that for patients with low $C X C R 7$ expression ( $P=0.019$, Figure 2).

\section{Prognostic value of CXCR7 expression in PCa}

Univariate and multivariate Cox regression analyses revealed a significant correlation between overall survival and $C X C R 7$ expression ( $\mathrm{HR}=2.271,95 \% \mathrm{Cl}=1.093-4.719, P=0.003$, Table 2$)$. Thus, $C X C R 7$ is a valuable independent biomarker for predicting the prognosis of PCa patients.

\section{Discussion}

PCa is a frequently diagnosed malignancy among men, with increasing morbidity with age $[15,16]$. PCa has a highly variable natural history. In patients diagnosed with indolent PCa, close surveillance is sufficient, while other patients need active treatments due to aggressive tumor progression [17]. It is challenging to discriminate between low-risk and highrisk PCa patients [18]. Prognostic biomarkers can accurately predict the progression of the cancer and can guide therapy. Conventionally, PCa prognosis evaluation is mainly based on surgical margin status, lymph node status, Gleason score, and PSA. Unfortunately, the prognostic performance of these factors is far from satisfactory $[17,19]$. Recently, in order to improve the management of $\mathrm{PCa}$, more and more molecular biomarkers have been confirmed for PCa. For examples, Deng et al. showed that serum $P C D H 10$ is an independent predictor for overall survival of PCa patients [20]. Cui et al. reported that SIRT1 can promote migration and invasion of PCa cells via epithelial-mesenchymal transition (EMT). SIRT1 might be a potential therapeutic target for PCa [21]. In addition to genes, the non-coding miRNAs might also take part in regulation of PCa. For instance, miR-301a and miR-301b were 2 hypoxia-responsive miRNAs that might be involved in radioresistance of PCa [22]. Accumulating evidence demonstrates that the PCarelated genes may provide new insights into the etiology of $\mathrm{PCa}$, which may be of great help in clinical practice.

Table 2. Univariable Cox analyses for $C X C R 7$ in 152 PCa patients.

\begin{tabular}{|c|c|c|c|c|c|c|}
\hline \multirow{2}{*}{ Characteristics } & \multicolumn{3}{|c|}{ Univariate analysis } & \multicolumn{3}{|c|}{ Multivariate analysis } \\
\hline & HR & $95 \% \mathrm{Cl}$ & $\boldsymbol{P}$ & HR & $95 \% \mathrm{Cl}$ & $\boldsymbol{P}$ \\
\hline CXCR7 (high vs. low) & 2.271 & $1.093-4.719$ & 0.028 & 2.271 & $1.093-4.719$ & 0.028 \\
\hline Age (year) ( $\geq 65$ vs. $<650$ & 1.399 & $0.752-2.602$ & 0.290 & - & - & - \\
\hline Tumor size $(\mathrm{cm})(\geq 3$ vs. $<3)$ & 1.002 & $0.555-1.809$ & 0.994 & - & - & - \\
\hline PSA (ng/ml) (>20 vs. $\leq 20)$ & 1.311 & $0.710-2.420$ & 0.386 & - & - & - \\
\hline Differentiation (poor vs. well+moderate) & 1.712 & $0.946-3.096$ & 0.075 & - & - & - \\
\hline TNM stage (III+IV vs. I+II) & 1.306 & $0.726-2.348$ & 0.373 & - & - & - \\
\hline Lymph node metastasis (positive vs. negative) & 1.215 & $0.675-2.188$ & 0.516 & - & - & - \\
\hline
\end{tabular}

'-' - indicated no related data. 
In recent years, $C X C R 7$ has been identified as a receptor to CXCL12, which is a 7-transmembrane span [23]. It was reported that dysregulation of $C X C R 7$ contributes to formation, invasion, and metastasis of cancers [24]. Elevated expression of $C X C R 7$ was observed in several cancers, such as lung, liver, brain, and breast cancers $[10,25,26]$. In this research, we investigated the CXCR7 expression pattern in PCa. QRT-PCR suggested that PCa tissues exhibited increased expression of $C X C R 7$ of $C X C R 7$ was significantly correlated with high serum level of PSA, poor differentiation, and positive lymph node metastasis. All the data revealed that $C X C R 7$ was an oncogene in $\mathrm{PCa}$ and promoted the malignant progression of $\mathrm{PCa}$.

In order to explore the oncogenic mechanisms of $C X C R 7$ in $P C a$, we performed a series of cell experiments. The results indicated that knockdown of CXCR7 significantly suppressed proliferation and migration of PCa cells. Based on the above results, we deduced that $C X C R 7$ was involved in the progression of $\mathrm{PCa}$ through regulating the proliferative and migratory abilities of the cancer cells. CXCR7 might be a therapeutic target for PCa. Mir et al. reported that Andrographolide damages $P C a$ cells via targeting the expression of CXCR3 and CXCR7 [27], supporting the conclusions of the present study. However, the specific molecular mechanisms by which CXCR7 affects $P C a$ remain unclear and further research is needed. compared with non-cancerous tissues. Moreover, upregulation

Given its functional roles in pathogenesis of malignancy, $C X C R 7$ is a predictive factor for several cancers. Wang et al. found that high expression of $C X C R 7$ predicted poor survival for patients with renal cell carcinoma [28]. In cervical cancer, upregulation of $C X C R 7$ was strongly correlated with aggressive clinical characteristics and might be an independent biomarker for disease-specific survival [29]. Yao et al. found that CXCR7 expression was closely associated with tumor stage, which could serve as an independent biomarker for gallbladder cancer patients [30]. In our study, the clinical prognosis value of $C X C R 7$ in PCa was evaluated. The results of Kaplan-Meier analysis showed that PCa patients in the high $C X C R 7$ expression group had poor survival rate. Moreover, Cox regression analysis further suggested that $C X C R 7$ is a valuable independent biomarker for the prognosis of PCa patients. In this study, we proved that high expression of $C X C R 7$ predicted poor prognosis for PCa patients. However, the sample size was relatively small in this study, and the utility of CXCR7 in PCa prognosis should be verified by further investigations.

\section{Conclusions}

Upregulation of $C X C R 7$ in PCa patients is significantly correlated with high concentration of PSA, poor differentiation, and positive metastasis. $C X C R 7$ may contribute to malignant progression of $\mathrm{PCa}$ by regulating the proliferative and migratory abilities of cancer cells, and CXCR7 may be an independent biomarker for prognosis of PCa patients.

\section{References:}

1. Siegel R, Naishadham D, Jemal A: Cancer statistics, 2012. Cancer J Clin, 2012; 62(1): 10-29

2. Center MM, Jemal A, Lortet-Tieulent J et al: International variation in prostate cancer incidence and mortality rates. Eur Urol, 2012; 61(6): 1079-92

3. Bao BY, Lin VC, Yu CC et al: Genetic variants in ultraconserved regions associate with prostate cancer recurrence and survival. Sci Rep, 2016; 6: 22124

4. Haldrup C, Lynnerup AS, Storebjerg TM et al: Large-scale evaluation of SLC18A2 in prostate cancer reveals diagnostic and prognostic biomarker potential at three molecular levels. Mol Oncol, 2016; 10(6): 825-37

5. Mukaida N, Sasaki S, Baba T: Chemokines in cancer development and progression and their potential as targeting molecules for cancer treatment Mediators Inflamm, 2014; 2014: 170381

6. Tsaur I, Noack A, Makarevic J et al: CCL2 chemokine as a potential biomarker for prostate cancer: A pilot study. Cancer Res Treat, 2015; 47(2): 306-12

7. Tang X, Li X, Li Z et al: Downregulation of CXCR7 inhibits proliferative Capacity and stem cell-like properties in breast cancer stem cells. Tumour Biol, 2016; 37(10): 13425-33

8. Balkwill F: Cancer and the chemokine network. Nat Rev Cancer, 2004; 4(7): 540-50

9. Miao Z, Luker KE, Summers BC et al: CXCR7 (RDC1) promotes breast and lung tumor growth in vivo and is expressed on tumor-associated vasculature. Proc Natl Acad Sci USA, 2007; 104(40): 15735-40

10. Hattermann K, Held-Feindt J, Lucius R et al: The chemokine receptor CXCR7 is highly expressed in human glioma cells and mediates antiapoptotic effects. Cancer Res, 2010; 70(8): 3299-308
11. Singh RK, Lokeshwar BL: The IL-8-regulated chemokine receptor CXCR7 stimulates EGFR signaling to promote prostate cancer growth. Cancer Res, 2011; 71(9): 3268-77

12. Edge SB, Compton CC: The American Joint Committee on Cancer: The $7^{\text {th }}$ edition of the AJCC cancer staging manual and the future of TNM. Ann Surg Oncol, 2010; 17(6): 1471-74

13. Epstein J, Zelefsky MJ, Sjoberg DD et al: A contemporary prostate cancer grading system: A validated alternative to the gleason score. Eur Urol, 2016; 69(3): 428-35

14. Yang D, Dai T, Xue L et al: Expression of chemokine receptor CXCR7 in colorectal carcinoma and its prognostic significance. Int J Clin Exp Pathol, 2015; 8(10): 13051-58

15. Siegel R, Naishadham D, Jemal A: Cancer statistics, 2013. Cancer J Clin, 2013; 63(1): 11-30

16. Ferlay J, Parkin DM, Steliarova-Foucher E: Estimates of cancer incidence and mortality in Europe in 2008. Eur J Cancer, 2010; 46(4): 765-81

17. Cai C, Chen JY, Han ZD et al: Down-regulation of dual-specificity phosphatase 5 predicts poor prognosis of patients with prostate cancer. Int J Clin Exp Med, 2015; 8(3): 4186-94

18. Martin NE: New developments in prostate cancer biomarkers. Curr Opin Oncol, 2016; 28(3): 248-52

19. Capitanio U, Briganti A, Gallina A et al: Predictive models before and after radical prostatectomy. Prostate, 2010; 70(12): 1371-78

20. Deng QK, Lei YG, Lin YL et al: Prognostic value of Protocadherin10 (PCDH10) methylation in serum of prostate cancer patients. Med Sci Monit, 2016; 22 516-21 
21. Ying Cui et al., Effect of SIRT1 Gene on Epithelial-Mesenchymal Transition of Human Prostate Cancer PC-3 Cells. Med Sci Monit, 2016; 22: 380-86

22. Cui Y, Li J, Zheng F et al: Hypoxia-responsive mir-301a and mir-301b promote radioresistance of prostate cancer cells via downregulating NDRG2. Med Sci Monit, 2016; 22: 2126-32

23. Sun X, Cheng G, Hao $M$ et al: CXCL12/CXCR4/CXCR7 chemokine axis and cancer progression. Cancer Metastasis Rev, 2010; 29(4): 709-22

24. Guo JC, Li J, Zhou L et al: CXCL12-CXCR7 axis contributes to the invasive phenotype of pancreatic cancer. Oncotarget, 2016; 7(38):62006-18

25. Burns JM, Summers BC, Wang Y et al: A novel chemokine receptor for SDF1 and I-TAC involved in cell survival, cell adhesion, and tumor development. J Exp Med, 2006; 203(9): 2201-13
26. Zabel BA, Lewén S, Berahovich RD et al: The novel chemokine receptor CXCR7 regulates trans-endothelial migration of cancer cells. Mol Cancer, 2011; 10: 73

27. Mir H, Kapur N, Singh R et al: Andrographolide inhibits prostate cancer by targeting cell cycle regulators, CXCR3 and CXCR7 chemokine receptors. Cell Cycle, 2016; 15(6): 819-26

28. Wang L, Chen W, Gao L et al: High expression of CXCR4, CXCR7 and SDF1 predicts poor survival in renal cell carcinoma. World J Surg Oncol, 2012; 10: 212

29. Nakhleh RE, Wesen C, Snover DC, Grage T: Venoocclusive lesions of the central veins and portal vein radicles secondary to intraarterial 5-fluoro-2'-deoxyuridine infusion. Hum Pathol, 1989; 20(12): 1218-20

30. Yao X, Zhou L, Han S, Chen Y: High expression of CXCR4 and CXCR7 predicts poor survival in gallbladder cancer. J Int Med Res, 2011; 39(4): 1253-64 